\title{
Common Fixed Point Theorem for Six Selfmaps of a Complete G-Metric Space
}

\author{
J. Niranjan Goud1, M. Rangamma² \\ ${ }^{1}$ Department of Mathematics, Government College for Men, Kurnool, India \\ ${ }^{2}$ Department of Mathematics, Osmania University, Hyderabad, India \\ Email: jngoud1979@gmail.com
}

How to cite this paper: Goud, J.N. and Rangamma, M. (2017) Common Fixed Point Theorem for Six Selfmaps of a Complete G-Metric Space. Advances in Pure Mathematics, 7, 290-297. https://doi.org/10.4236/apm.2017.73015

Received: January 24, 2017

Accepted: March 28, 2017

Published: March 31, 2017

Copyright $\odot 2017$ by authors and Scientific Research Publishing Inc. This work is licensed under the Creative Commons Attribution International License (CC BY 4.0).

http://creativecommons.org/licenses/by/4.0/

\begin{abstract}
By using weakly compatible conditions of selfmapping pairs, we prove a common fixed point theorem for six mappings in generalized complete metric spaces. An example is provided to support our result.
\end{abstract}

\section{Keywords}

G-Metric Space, Weakly Compatible Mappings, Fixed Point, Associated Sequence of a Point Relative to Six Selfmaps

\section{Introduction}

The study of fixed point theory has been at the centre of vigorous activity and it has a wide range of applications in applied mathematics and sciences. Over the past two decades, a considerable amount of research work for the development of fixed point theory have executed by several authors.

In 1963, Gahler [1] [2] introduced 2-metric spaces and claimed them as generalizations of metric spaces. But many researchers proved that there was no relation between these two spaces. These considerations led Dhage [3] to initiate a study of general metric spaces called D-metric spaces. As a probable modification to D-metric spaces, Shaban Sedghi, Nabi Shobe and Haiyun Zhou [4] have introduced $D^{*}$-metric spaces. In 2006, Zead Mustafa and Brailey Sims [5] initiated $G$-metric spaces. Several researchers proved many common fixed point theorems on $G$-metric spaces.

The purpose of this paper is to prove a common fixed point theorem for six weakly compatible selfmaps of a complete $G$-metric space. Now we recall some basic definitions and results on $G$-metric space.

\section{Preliminaries}

We begin with 
Definition 2.1: ([5], Definition 3) Let $X$ be a non-empty set and $G: X^{3} \rightarrow[0, \infty)$ be a function satisfying:

(G1) $G(x, y, z)=0$ if $x=y=z$.

(G2) $0<G(x, x, y)$ for all $x, y \in X$ with $x \neq y$.

(G3) $G(x, x, y)<G(x, y, z)$ for all $x, y, z \in X$ with $y \neq z$.

(G4) $G(x, y, z)=G(\sigma(x, y, z))$ for all $x, y, z \in X$, where $\sigma(x, y, z)$ is a permutation of the set $\{x, y, z\}$.

And

(G5) $G(x, y, z)<G(x, w, w)+G(w, y, z)$ for all $x, y, z, w \in X$.

Then $G$ is called a $G$-metric on $X$ and the pair $(X, G)$ is called a $G$-metric Space.

Definition 2.2: ([5], Definition 4) A $G$-metric Space $(X, G)$ is said to be symmetric if

(G6) $G(x, y, y)=G(x, x, y)$ for all $x, y \in X$.

The example given below is a non-symmetric $G$-metric space.

Example 2.3: ([5], Example 1): Let $X=\{a, b\}$ Define $G: X^{3} \rightarrow[0, \infty)$ by

$G(a, a, a)=G(b, b, b)=0 ; \quad G(a, a, b)=1, G(a, b, b)=2$ and extend $G$ to all of $X^{3}$ by using (G4).

Then it is easy to verify that $(X, G)$ is a $G$-metric space. Since $G(a, a, b) \neq G(a, b, b)$, the space $(X, G)$ is non-symmetric, in view of (G6).

Example 2.4: Let $(X, d)$ be a metric space. Define $G_{s}^{d}: X^{3} \rightarrow[0, \infty)$ by $G_{s}^{d}(x, y, z)=\frac{1}{3}[d(x, y)+d(y, z)+d(z, x)]$ for $x, y, z \in X$.Then $\left(X, G_{s}^{d}\right)$ is a $G$-metric Space.

Lemma (2.5): ([5], p. 292) If $(X, G)$ is a $G$-metric space then $G(x, y, y) \leq 2 G(y, x, x)$ for all $x, y \in X$.

Definition 2.6: Let $(X, G)$ be a $G$-metric Space. A sequence $\left\{x_{n}\right\}$ in $X$ is said to be $G$-convergent if there is a $x_{0} \in X$ such that to each $\varepsilon>0$ there is a natural number $N$ for which $G\left(x_{n}, x_{n}, x_{0}\right)<\varepsilon$ for all $n \geq N$.

Lemma 2.7: ([5], Proposition 6) Let $(X, G)$ be a $G$-metric Space, then for a sequence $\left\{x_{n}\right\} \subseteq X$ and point $x \in X$ the following are equivalent.

(1) $\left\{x_{n}\right\}$ is $G$ - convergent to $x$.

(2) $d_{G}\left(x_{n}, x\right) \rightarrow 0$ as $n \rightarrow \infty$ (that is $\left\{x_{n}\right\}$ converges to $x$ relative to the metric $\left.d_{G}\right)$.

(3) $G\left(x_{n}, x_{n}, x\right) \rightarrow 0$ as $n \rightarrow \infty$.

(4) $G\left(x_{n}, x, x\right) \rightarrow 0$ as $n \rightarrow \infty$.

(5) $G\left(x_{m}, x_{n}, x\right) \rightarrow 0$ as $m, n \rightarrow \infty$.

Definition 2.8: ([5], Definition 8) Let $(X, G)$ be a $G$-metric space, then a sequence $\left\{x_{n}\right\} \subseteq X$ is said to be $G$-Cauchy if for each $\varepsilon>0$, there exists a natural number $N$ such that $G\left(x_{n}, x_{m}, x_{l}\right)<\varepsilon$ for all $n, m, l \geq N$.

Note that every $G$-convergent sequence in a $G$-metric space $(X, G)$ is $G$ Cauchy.

Definition 2.9: ([5], Definition 9) A $G$-metric space $(X, G)$ is said to be $G$ complete if every $G$-Cauchy sequence in $(X, G)$ is $G$-convergent in $(X, G)$.

Gerald Jungck [6] initiated the notion of weakly compatible mappings, as a 
generalization of commuting maps. We now give the definition of weakly compatibility in a $G$-metric space.

Definition 2.10: [7] Suppose $f$ and $g$ are selfmaps of a $G$-metric space $(X, G)$. The pair $(f, g)$ is said to be weakly compatible if $G(f g x, g f x, g f x)=0$ whenever $G(f x, g x, g x)=0$.

\section{Main Theorem}

Theorem 3.1: Suppose $f, g, h, p, Q$ and $R$ are six selfmaps of a complete $G$ -metric space $(X, G)$ satisfying the following conditions.

(3.1.1) $f g(X) \subseteq R(X)$ and $h p(X) \subseteq Q(X)$,

$$
\begin{aligned}
G(h p x, f g y, f g y) & \leq \alpha G(R x, Q y, Q y)+\beta[G(R x, h p x, h p x)+G(Q y, f g y, f g y)] \\
& +\gamma[G(R x, f g y, f g y)+G(h p x, Q y, Q y)]
\end{aligned}
$$

for all $x, y \in X$ and $\alpha, \beta, \gamma$ are non-negative real numbers such that $\alpha+2 \beta+2 \gamma<1$,

(3.1.3) one of $R(X), Q(X)$ is closed sub subset of $X$,

(3.1.4) $(f g, Q)$ and $(h p, R)$ are weakly compatible pairs,

(3.1.5) The pairs $(h, p),(h, R),(f, g)$, and $(f, Q)$ are commuting.

Then $f, g, h, p, Q$ and $R$ have a unique common fixed point in $X$.

Proof: Let $x_{0} \in X$ be an arbitrary point. Since $f g(X) \subseteq R(X)$ and $h p(X) \subseteq Q(X)$ there exists $x_{1}, x_{2} \in X$ such that $h p x_{0}=Q x_{1}$ and $f g x_{1}=R x_{2}$ again there exists $x_{3}, x_{4} \in X$ such that $h p x_{2}=Q x_{3}$ and $f g x_{3}=R x_{4}$, continuing in the same manner for each $n \geq 0$, we obtain a sequence $\left\{x_{n}\right\}$ in $X$ such that

$$
y_{2 n}=h p x_{2 n}=Q x_{2 n+1}, \quad y_{2 n+1}=f g x_{2 n+1}=R x_{2 n+2} \text { for } n \geq 0 .
$$

From condition (3.1.2), we have

$$
\begin{aligned}
G\left(y_{2 n}, y_{2 n+1}, y_{2 n+1}\right) & =G\left(h p x_{2 n}, f g x_{2 n+1}, f g x_{2 n+1}\right) \\
\leq & \alpha G\left(R x_{2 n}, Q x_{2 n+1}, Q x_{2 n+1}\right)+\beta\left[G\left(R x_{2 n}, h p x_{2 n}, h p x_{2 n}\right)+G\left(Q x_{2 n+1}, f g x_{2 n+1}, f g x_{2 n+1}\right)\right] \\
& +\gamma\left[G\left(R x_{2 n}, f g x_{2 n+1}, f g x_{2 n+1}\right)+G\left(h p x_{2 n}, Q x_{2 n+1}, Q x_{2 n+1}\right)\right] \\
& =\alpha G\left(y_{2 n-1}, y_{2 n}, y_{2 n}\right)+\beta\left[G\left(y_{2 n-1}, y_{2 n}, y_{2 n}\right)+G\left(y_{2 n}, y_{2 n+1}, y_{2 n+1}\right)\right] \\
& +\gamma\left[G\left(y_{2 n-1}, y_{2 n+1}, y_{2 n+1}\right)+G\left(y_{2 n}, y_{2 n}, y_{2 n}\right)\right] \\
\leq & (\alpha+\beta+\gamma) G\left(y_{2 n-1}, y_{2 n}, y_{2 n}\right)+(\beta+\gamma) G\left(y_{2 n}, y_{2 n+1}, y_{2 n+1}\right) .
\end{aligned}
$$

Therefore

$$
\begin{gathered}
(1-\beta-\gamma) G\left(y_{2 n}, y_{2 n+1}, y_{2 n+1}\right) \leq(\alpha+\beta+\gamma) G\left(y_{2 n-1}, y_{2 n}, y_{2 n}\right) \\
G\left(y_{2 n}, y_{2 n+1}, y_{2 n+1}\right) \leq \frac{(\alpha+\beta+\gamma)}{(1-\beta-\gamma)} G\left(y_{2 n-1}, y_{2 n}, y_{2 n}\right) \\
G\left(y_{2 n}, y_{2 n+1}, y_{2 n+1}\right) \leq k G\left(y_{2 n-1}, y_{2 n}, y_{2 n}\right)
\end{gathered}
$$

where $k=\frac{(\alpha+\beta+\gamma)}{(1-\beta-\gamma)}<1$.

Similarly, we can show that 


$$
G\left(y_{2 n+1}, y_{2 n+2}, y_{2 n+2}\right) \leq k G\left(y_{2 n}, y_{2 n+1}, y_{2 n+1}\right) .
$$

From (3.1.7) and (3.1.8) we have

$$
G\left(y_{n}, y_{n+1}, y_{n+1}\right) \leq k G\left(y_{n-1}, y_{n}, y_{n}\right) \leq \cdots \leq k^{n} G\left(y_{0}, y_{1}, y_{1}\right) .
$$

Now for every $n, m \in N$ such that $m>n$ we have

$$
\begin{aligned}
G\left(y_{n}, y_{m}, y_{m}\right) & \leq G\left(y_{n}, y_{n+1}, y_{n+1}\right)+G\left(y_{n+1}, y_{n+2}, y_{n+2}\right)+\cdots+G\left(y_{m-1}, y_{m}, y_{m}\right) \\
& \leq k^{n} G\left(y_{0}, y_{1}, y_{1}\right)+k^{n+1} G\left(y_{0}, y_{1}, y_{1}\right)+\cdots+k^{m-1} G\left(y_{0}, y_{1}, y_{1}\right) \\
& \leq k^{n}\left(1+k+k^{2}+\cdots+k^{m-n+1}\right) G\left(y_{0}, y_{1}, y_{1}\right) \\
& \leq k^{n} \frac{\left(1-k^{m-n}\right)}{1-k} G\left(h x_{0}, h x_{1}, h x_{1}\right) \rightarrow 0 \text { as } n \rightarrow \infty .
\end{aligned}
$$

Since $k<1$.

Therefore, $\left\{y_{n}\right\}$ is a Cauchy sequence in $X$. Since $X$ is a complete $G$-metric space, then there exists a point $z \in X$ such that

$$
\lim _{n \rightarrow \infty} h p x_{2 n}=\lim _{n \rightarrow \infty} Q x_{2 n+1}=\lim _{n \rightarrow \infty} f g x_{2 n+1}=\lim _{n \rightarrow \infty} R x_{2 n+2}=z .
$$

If $R(X)$ is a closed subset of $X$, then there exists a point $u \in X$ such that $\mathrm{z}=\mathrm{Ru}$.

Now from (3.1.2), we have

$$
\begin{aligned}
G\left(h p u, f g x_{2 n+1}, f g x_{2 n+1}\right) & \leq \alpha G\left(R u, Q x_{2 n+1}, Q x_{2 n+1}\right)+\beta\left[G(R u, h p u, h p u)+G\left(Q x_{2 n+1}, f g x_{2 n+1}, f g x_{2 n+1}\right)\right] \\
& +\gamma\left[G\left(R u, f g x_{2 n+1}, f g x_{2 n+1}\right)+G\left(h p u, Q x_{2 n+1}, Q x_{2 n+1}\right)\right] .
\end{aligned}
$$

Letting $n \rightarrow \infty$ in (3.1.10) and by the continuity of $G$ we have

$$
\begin{aligned}
G(h p u, z, z) \leq & \alpha G(z, z, z)+\beta[G(z, h p u, h p u)+G(z, z, z)] \\
& +\gamma[G(z, z, z)+G(h p u, z, z)] \\
\leq & (2 \beta+\gamma) G(h p u, z, z),
\end{aligned}
$$

which leads to a contradiction as $2 \beta+\gamma<1$.

Hence $G(h p u, z, z)=0$, which implies $h p u=z$.

Therefore,

$$
h p u=R u=z .
$$

Now since $h p(X) \subseteq Q(X)$ then there exists a point $v \in X$ such that $Z=Q v$.

Then we have by (3.1.2)

$G(h p u, f g v, f g v) \leq \alpha G(R u, Q v, Q v)+\beta[G(R u, h p u, h p u)+G(Q v, f g v, f g v)]$

$$
\begin{aligned}
+\gamma[G(R u, f g v, f g v)+G(h p u, Q v, Q v)] \\
\begin{aligned}
G(z, f g v, f g v) \leq & \alpha G(z, z, z)+\beta[G(z, z, z)+G(z, f g v, f g v)] \\
& +\gamma[G(z, f g v, f g v)+G(z, z, z)] \\
\leq & (\beta+\gamma) G(z, f g v, f g v),
\end{aligned}
\end{aligned}
$$

which leads to a contradiction, since $\beta+\gamma<1$. Hence $f g v=z$.

Therefore,

$$
f g v=Q v=z
$$


From (3.1.11) and (3.1.13) we have $R u=h p u=f g v=Q v=z$.

Since the pair $(f g, Q)$ is weakly compatible then $f g Q v=Q f g v$ which gives $f g z=Q z$.

Now (3.1.2) we have

$$
\begin{aligned}
G(z, f g z, f g z)= & G(h p u, f g z, f g z) \\
\leq & \alpha G(R u, Q z, Q z)+\beta[G(R u, h p u, h p u)+G(Q z, f g z, f g z)] \\
& +\gamma[G(R u, f g z, f g z)+G(h p u, Q z, Q z)] \\
= & \alpha G(z, f g z, f g z)+\beta[G(z, z, z)+G(f g z, f g z, f g z)] \\
& +\gamma[G(z, f g z, f g z)+G(z, f g z, f g z)] \\
= & (\alpha+2 \gamma) G(z, f g z, f g z)
\end{aligned}
$$

which is a contradiction, since $\alpha+2 \gamma<1$. Hence $G(z, f g z, f g z)=0$ thus $f g z=z$.

Showing that $z$ is a common fixed point of $f g$ and $Q$.

Since the pair $(h p, R)$ is weakly compatible then $h p R u=R h p u$ which gives $h p z=R z$.

Then we have by (3.1.2)

$$
\begin{aligned}
G(h p z, z, z)= & G(h p z, f g z, f g z) \\
\leq & \alpha G(R z, Q z, Q z)+\beta[G(R z, h p z, h p z)+G(Q z, f g z, f g z)] \\
& +\gamma[G(R z, f g z, f g z)+G(h p z, Q z, Q z)] \\
= & \alpha G(h p z, z, z)+\beta[G(h p z, h p z, h p z)+G(z, z, z)] \\
& +\gamma[G(h p z, z, z)+G(h p z, z, z)] \\
= & (\alpha+2 \gamma) G(h p z, z, z),
\end{aligned}
$$

which is a contradiction, since $\alpha+2 \gamma<1$. Hence $G(h p z, z, z)=0$ thus $h p z=z$.

Showing that $z$ is a common fixed point of $h p$ and $R$.

Therefore, $z$ is a common fixed point of $f g, h p, R$ and $Q$.

By commuting conditions of the pairs in (3.1.5), we have

$$
f z=f(f g z)=f(g f z)=f g(f z), \quad f z=f(Q z)=Q(f z) .
$$

And

$$
h z=h(h p z)=h(p h z)=h p(h z), \quad h z=h(R z)=R(h z) .
$$

From (3.1.2)

$$
\begin{aligned}
G(z, f z, f z)= & G(h p z, f g f z, f g f z) \\
\leq & \alpha G(R z, Q f z, Q f z)+\beta[G(R z, h p z, h p z)+G(Q f z, f g f z, f g f z)] \\
& +\gamma[G(R z, f g f z, f g f z)+G(h p z, Q f z, Q f z)] \\
= & \alpha G(z, f z, f z)+\beta[G(z, z, z)+G(f z, f z, f z)] \\
& +\gamma[G(z, f z, f z)+G(z, f z, f z)] \\
= & (\alpha+2 \gamma) G(z, f z, f z) .
\end{aligned}
$$

Since $\alpha+2 \gamma<1$, we have $G(z, f z, f z)=0$ thus $f z=z$.

Also $g z=g f z=f g z=z$.

Therefore, we have $f z=g z=R z=f g z=z$. 
Similarly, we have $h z=p z=Q z=h p z=z$.

Therefore, $z$ is a common fixed point of $f, g, h, p, Q$ and $R$.

The proof is similar in case if $Q(X)$ is a closed subset of $X$.

We now prove the uniqueness of the common fixed point.

If possible, assume that $w$ is another common fixed point of $f, g, h, p, Q$ and $R$.

By condition (3.1.2) we have

$$
\begin{aligned}
G(z, w, w)= & G(h p z, f g w, f g w) \\
\leq & \alpha G(R z, Q w, Q w)+\beta[G(R z, h p z, h p z)+G(Q w, f g w, f g w)] \\
& +\gamma[G(R z, f g w, f g w)+G(h p z, Q w, Q w)] \\
= & \alpha G(z, w, w)+\beta[G(z, z, z)+G(w, w, w)]+\gamma[G(z, w, w))+G(z, w, w)] \\
= & (\alpha+2 \gamma) G(z, w, w),
\end{aligned}
$$

which is a contradiction, since $\alpha+2 \gamma<1$.

Hence $G(z, w, w)=0$ which gives $z=w$.

Therefore, $Z$ is a unique common fixed point of $f, g, h, p, Q$ and $R$.

As an example, we have the following.

\subsection{Example}

Let $X=[0,1]$ with $G(x, y, z)=|x-y|+|y-z|+|z-x|$ for $x, y, z \in X$. Then $\mathrm{G}$ is a G-metric on $X$.

Define

$$
f: X \rightarrow X, g: X \rightarrow X, h: X \rightarrow X, p: X \rightarrow X, Q: X \rightarrow X, R: X \rightarrow X
$$

by

$$
\begin{gathered}
f x=h x=\frac{x+1}{3}, \forall x \in X, \\
g x=p x=\frac{3 x+1}{5}, \forall x \in X, \\
Q x=R x=x, \forall x \in X . \\
f g x=f\left(\frac{3 x+1}{5}\right)=\frac{x+2}{5}, h p x=h\left(\frac{3 x+1}{5}\right)=\frac{x+2}{5}, \\
f g X=\left[\frac{2}{5}, \frac{3}{5}\right], h p X=\left[\frac{2}{5}, \frac{3}{5}\right], R X=[0,1], Q X=[0,1] \\
f g X \subseteq R X, h p X \subseteq Q X .
\end{gathered}
$$

Proving the condition (3.1.1) of the Theorem (3.1).

$R X$ and $Q X$ are closed subsets of $X$. Proving the condition (3.1.3) of the Theorem (3.1).

Since $f g\left(\frac{1}{2}\right)=\frac{1}{2}$ and $Q\left(\frac{1}{2}\right)=\frac{1}{2}$ then $f g Q\left(\frac{1}{2}\right)=Q f g\left(\frac{1}{2}\right)$, showing that the pair $(f g, Q)$ is weakly compatible.

Also, the pair $(h p, R)$ is weakly compatible.

Proving the condition (3.1.4) of the Theorem (3.1). 


$$
\begin{aligned}
& h p(x)=\frac{x+2}{5}=p h(x), h R(x)=h(x)=R h(x), \\
& f g(x)=\frac{x+2}{5}=g f(x), f Q(x)=f(x)=Q f(x),
\end{aligned}
$$

showing that $(h, R),(f, Q),(h, p)$ and $(f, g)$ are commuting pairs.

Proving the condition (3.1.5) of the Theorem (3.1).

Now we prove the condition (3.1.2) of the Theorem (3.1).

On taking $\alpha=\frac{1}{10}, \beta=\frac{1}{8}, \gamma=\frac{1}{12}$ then $\alpha+2 \beta+2 \gamma=\frac{31}{60}<1$.

Now $G(h p x, f g y, f g y)=2|h p x-f g y|=\frac{2}{5}|x-y|$

$$
\begin{gathered}
G(R x, Q y, Q y)=2|R x-Q y|=2|x-y|, \\
G(R x, h p x, h p x)=2|R x-h p x|=\frac{4}{5}|2 x-1|, \\
G(Q y, f g y, f g y)=2|f g y-Q y|=\frac{4}{5}|1-2 y|, \\
G(R x, f g y, f g y)=2|R x-f g y|=\frac{2}{5}|5 x-y-2|, \\
G(h p x, Q y, Q y)=2|h p x-Q y|=\frac{2}{5}|x+2-5 y| \\
+\gamma G(R x, Q y, Q y)+\beta[G(R x, h p x, h p x)+G(Q y, f g y, f g y)] \\
=2 \alpha|x-y|+\frac{4}{5} \beta(|2 x-1|+|1-2 y|)+\frac{2}{5} \gamma(|5 x-y-2|+|x-5 y-2|) \\
\geq 2 \alpha|x-y|+\frac{4}{5} \beta|2 x-2 y|+\frac{2}{5} \gamma|6 x-6 y| \\
=\left(2 \alpha+\frac{8 \beta}{5}+\frac{12}{5} \gamma\right)|x-y| \\
=\frac{3}{5}|x-y| \geq \frac{2}{5}|x-y|=G(f g x, h p y, h p y) .
\end{gathered}
$$

Therefore,

$G(h p x, f g y, f g y) \leq \alpha G(R x, Q y, Q y)+\beta[G(R x, h p x, h p x)+G(Q y, f g y, f g y)]$

$$
+\gamma[G(R x, f g y, f g y)+G(h p x, Q y, Q y)] .
$$

Proving the condition (3.1.2) of the Theorem (3.1).

Hence all the conditions of the Theorem (3.1) are satisfied.

Therefore, $\frac{1}{2}$ is a unique common fixed point of $f, g, h, p, Q$ and $R$.

\subsection{Corollary}

Suppose $f, p, Q$ and $R$ are four selfmaps of a complete $G$-metric space $(X, G)$ satisfying the following conditions:

(3.1.1) $f(X) \subseteq R(X)$ and $p(X) \subseteq Q(X)$,

$$
\begin{aligned}
G(p x, f y, f y) \leq & \alpha G(R x, Q y, Q y)+\beta[G(R x, p x, p x)+G(Q y, f y, f y)] \\
& +\gamma[G(R x, f y, f y)+G(p x, Q y, Q y)]
\end{aligned}
$$


for all $x, y \in X$ and $\alpha, \beta, \gamma$ are non-negative real numbers such that $\alpha+2 \beta+2 \gamma<1$,

(3.1.3) One of $R(X), Q(X)$ is closed sub subset of $X$,

(3.1.4) $(p, R)$ and $(f, Q)$ are weakly compatible pairs,

Then $f, p, Q$ and $R$ have a unique common fixed point in $X$.

Proof: Follows from the Theorem (3.1) if $g=h=I$ the identity map.

\subsection{Corollary}

Suppose $f, p$ and $R$ are three selfmaps of a complete $G$-metric space $(X, G)$ satisfying the following conditions:

(3.1.1) $f(X) \subseteq R(X)$ and $p(X) \subseteq R(X)$,

$$
\begin{aligned}
G(p x, f y, f y) \leq & \alpha G(R x, R y, R y)+\beta[G(R x, p x, p x)+G(R y, f y, f y)] \\
& +\gamma[G(R x, f y, f y)+G(p x, R y, R y)]
\end{aligned}
$$

for all $x, y \in X$ and $\alpha, \beta, \gamma$ are non-negative real numbers such that $\alpha+2 \beta+2 \gamma<1$,

(3.1.3) $R(X)$ is closed sub subset of $X$,

(3.1.4) $(p, R)$ and $(f, R)$ are weakly compatible pairs.

Then $f, p$ and $R$ have a unique common fixed point in $X$.

Proof: Follows from the Theorem (3.1) if $g=h=I$ the identity map, and $Q=R$.

\section{References}

[1] Gahler, S. (1963) 2-Metriche Raume and Ihre Topologische Strukture. Mathematische Nachrichten, 26, 115-148. https://doi.org/10.1002/mana.19630260109

[2] Gahler, S. (1966) Zur Geometric 2-Metriche Raume. Reevue Roumaine de math, 11, 664-669.

[3] Dhage, B.C. (1992) Generalized Metric Space and Mapping with Fixed Point. Bull.Cal.Math.Soc., 84, 329-336.

[4] Sedgi, S., Shobe, N. and Zhou, H.Y. () A Common Fixed Point Theorem in $D^{*}$ -Metric Space. Journal of Fixed Point Theory and Applications, 2007, 1-13.

[5] Mustafa, Z. and Sims, B. (2006) A New Approach to Generalize Metric Space. Journal of Non Linear and Convex Analysis, 2, 289-297.

[6] Jungck, G. (1996) Common Fixed Points for Non-Continuous Nonselfmaps on Non Metricspaces. Far East J.Math. Sci, 4, 199-215.

[7] Niranjan Goud, J. and Rangamma, M. (2016) A Common Fixed Point Theorem for Four Weakly Compatible Selfmaps of a Complete G-Metric Space. IJMA, 7, 104110. 
Submit or recommend next manuscript to SCIRP and we will provide best service for you:

Accepting pre-submission inquiries through Email, Facebook, LinkedIn, Twitter, etc. A wide selection of journals (inclusive of 9 subjects, more than 200 journals)

Providing 24-hour high-quality service

User-friendly online submission system

Fair and swift peer-review system

Efficient typesetting and proofreading procedure

Display of the result of downloads and visits, as well as the number of cited articles Maximum dissemination of your research work

Submit your manuscript at: http://papersubmission.scirp.org/

Or contact apm@scirp.org 